\title{
Téoros
}

Revue de recherche en tourisme

\section{Panorama et analyse descriptive de la fréquentation touristique européenne en Languedoc-Roussillon}

\section{Nicolas Peypoch et Bernardin Solonandrasana}

Volume 25, numéro 1, printemps 2006

URI : https://id.erudit.org/iderudit/1071035ar

DOI : https://doi.org/10.7202/1071035ar

Aller au sommaire du numéro

Éditeur(s)

Université du Québec à Montréal

ISSN

0712-8657 (imprimé)

1923-2705 (numérique)

Découvrir la revue

Citer cet article

Peypoch, N. \& Solonandrasana, B. (2006). Panorama et analyse descriptive de la fréquentation touristique européenne en Languedoc-Roussillon. Téoros, 25(1), 50-54. https://doi.org/10.7202/1071035ar d'utilisation que vous pouvez consulter en ligne. 


\section{Panorama et analyse descriptive de la fréquentation touristique européenne en Languedoc-Roussillon}

\section{Nicolas Peypoch et Bernardin Solonandrasana ${ }^{1}$}

\section{Introduction}

L'industrie du tourisme est une des plus importantes au monde et celle qui génère le plus d'emplois. Par ailleurs, la France est le pays le plus visité au monde. La région du Languedoc-Roussillon (L-R), quant à elle, présente de nombreux atouts touristiques avec son littoral, son espace montagne et ses nombreux sites remarquables. En 2003, le $\mathrm{L}-\mathrm{R}$ est la troisième région touristique française (hors Île-de-France) après les régions Rhône-Alpes et Provence-Alpes-Côte d'Azur. Elle enregistre 15 millions d'arrivées de touristes dont 4 millions d'étrangers, ce qui représente 103 millions de nuitées, dont $40 \%$ sont enregistrées hors des mois de juillet et d'août. Du côté de l'offre, 2 millions de lits sont proposés, ce qui représente quasiment un lit touristique pour un habitant de la région; en effet, la région compte 2,3 millions d'habitants. Le L-R possède également la durée moyenne de séjour la plus élevée des régions françaises avec 7,5 nuitées, tous hébergements confondus. Sur le plan de l'emploi, le tourisme représente 65000 actifs directs dont 32000 saisonniers, soit l'équivalent du secteur du bâtiment ou de l'agroalimentaire de la région. En matière de consommation, ajoutons que les touristes dépensent 4,5 à 5 milliards d'euros dans la région, soit l'équivalent de 10,5\% du produit intérieur brut (PIB) régional. Et, en termes d'arrivées, si la région L-R était un pays, elle arriverait en trentième position mondiale devant des pays comme le Maroc ou la Tunisie 2 .

Ces bons résultats ne sont pas les fruits du hasard. En effet, depuis la «Mission Racine» (mission interministérielle d'aménagement touristique présidée par P. Racine) qui a engagé l'aménagement de plusieurs stations balnéaires, comme Port-Camargue et $\mathrm{La}$ Grande Motte, sur le littoral languedocien, la région L-R a toujours été l'une des premières destinations de la côte méditerranéenne (Racine, 1980). Dans les années 1990, plus précisément en 1992, elle a essayé d'adapter ses offres et ses stratégies dans le but d'améliorer les résultats de son secteur touristique (Klemm, 1996). Bien que ces résultats soient très impressionnants, la part des touristes européens reste stable, voire légèrement en baisse sur la période 1993 à 2003. Une vision d'ensemble du comportement de ces touristes dans la région nous semble donc importante afin d'apprécier et de mieux accompagner ce changement de stratégies.

Nous nous concentrons ici sur les quatre nationalités qui forment le peloton de tête des fréquentations touristiques de la région, à savoir : les Allemands, les Anglais, les Belges et les Hollandais, car les données montrent que, sur cette période, bien que la fréquentation ait augmenté de $18 \%$, la part des touristes européens a baissé, passant de $29 \%$ en 1993 à 26,6 \% en 2003.

L'objet de ce travail est double. Dans un premier temps, nous allons dresser un panorama de la fréquentation touristique européenne en $L-R$ en analysant la durée des séjours ainsi que les comportements des touristes. La sophistication et la complexité des données nous conduisent à une analyse graphique retraçant l'évolution des nuitées et des arrivées par nationalité et par type d'hébergement. Nous procéderons ensuite à une étude détaillée de la relation entre l'évolution du nombre de nuitées et d'arrivées, selon le type d'hébergement. Enfin, nous affinerons chaque cas en introduisant les différentes nationalités de ces touristes. Cela nous permettra de proposer une analyse détaillée et précise de l'ensemble des données afin de mieux cerner la durée des séjours touristiques des européens en L-R. Dans un second temps, nous compléterons cette étude par une réflexion autour du comportement de ces touristes à travers leur conception des « attractions " proposées par la région. En effet, une analyse en termes d'attractions peut permettre de prévoir une éventuelle répétition de séjours chez les touristes (Peypoch et al., 2005 ; Peypoch et Solonandrasana, à paraitre).

\section{Analyse de la durée des séjours en Languedoc-Roussillon}

Cette première partie est consacrée à une analyse descriptive détaillée de la durée des séjours touristiques. Pour ce faire, nous alIons considérer différents éléments, à savoir le type d'hébergement, la nationalité des touristes et la durée des séjours des touristes puisque nous croyons que seul un découpage minutieux de la durée des séjours nous permettra de dégager le véritable comportement du touriste. Nous adopterons donc le concept d'analyse pyramidale ascendante : il s'agit de débuter par une description globale et générale du phénomène pour terminer par une analyse descriptive détaillée. Par conséquent, nous analyserons d'abord les séjours touristiques selon le type d'hébergement (hôtel et camping), ce qui nous permettra de dégager des tendances générales. Nous affinerons ensuite notre analyse en considérant les différentes nationalités des touristes, tout en conservant le type d'hébergement, ce qui nous permettra de mettre en évidence le comportement des touristes selon de multiples critères. 


\section{Analyse de la durée des séjours touristiques selon le type d'hébergement}

En matière d'hébergement, nous privilégions les hôtels et les campings et nous choisissons d'analyser la durée des séjours touristiques en L-R, de 1993 à 2003, par l'intermédiaire des rapports entre les nombres de nuitées et d'arrivées. Ainsi, les nuitées dans les résidences secondaires et chez les amis n'étant pas disponibles sous forme de données, elles ne sont pas prises en compte.

Nous représentons dans les graphiques $1 \mathrm{a}$ et $1 \mathrm{~b}$ les évolutions des arrivées et des nuitées dans les campings et les hôtels pour la période à l'étude.

Nous interprétons ces graphiques en privilégiant l'évolution du nombre d'arrivées. En effet, les observations qui suivent sont également valables lorsque nous considérons l'évolution du nombre de nuitées.

D’une manière générale, nous remarquons que, pour ces deux types d'hébergement, les évolutions du nombre d'arrivées sont similaires : nous constatons une baisse jusqu'en 1996, année qui marque le début d'une phase de croissance continue jusqu'en 2001. Ensuite, la tendance est à nouveau à la baisse. Le creux significatif constaté dans les deux graphiques précédents (1a et 1b) peut s'expliquer par des raisons bien connues à présent, à savoir : la grève des routiers de 1995 ; la dévaluation des monnaies italienne et espagnole (les deux pays qui sont considérés comme les deux concurrents directs de la France, notamment par leur situation géographique et leur patrimoine culturel...), rendant ces pays plus attractifs; et enfin la déréglementation des transports aériens. Ces phénomènes ont touché tout particulièrement le $L-R$ en raison de sa proximité avec I'Espagne. Ce dernier pays est, en effet, devenu un "bien substituable" (Bordet et al., 2004) aux yeux des touristes, réduisant ainsi la région à un simple lieu de passage. Quant à la baisse observée à partir de 2001, nous pouvons l'attribuer, d'une part, à la mise en circulation de l'euro offrant une meilleure lisibilité et permettant en conséquence une comparaison des prix et, d'autre part, à l'élargissement européen, entraînant ainsi un éventail élargi de destinations touristiques (la Croatie par exemple). En effet, un certain nombre de nouvelles

\section{Graphique 1a}

\section{Évolution des arrivées des touristes européens}

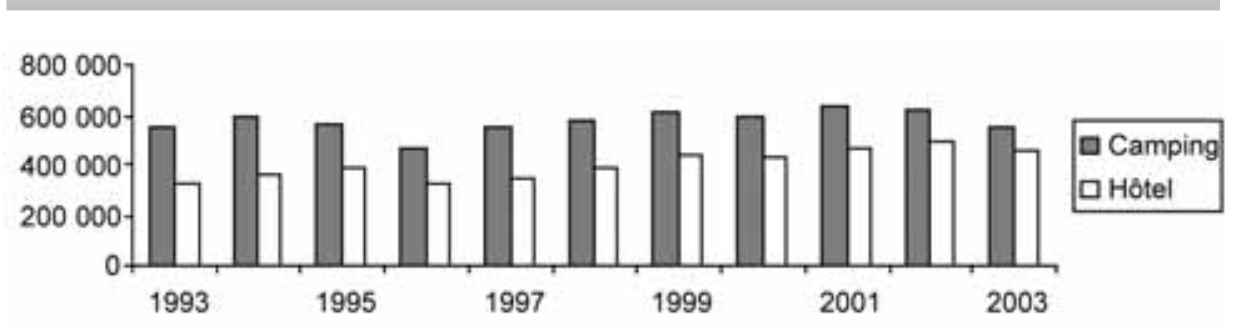

Source : Observatoire régional du tourisme du Languedoc-Roussillon (2004).

\section{Graphique 1b}

\section{Évolution des nuitées des touristes européens}

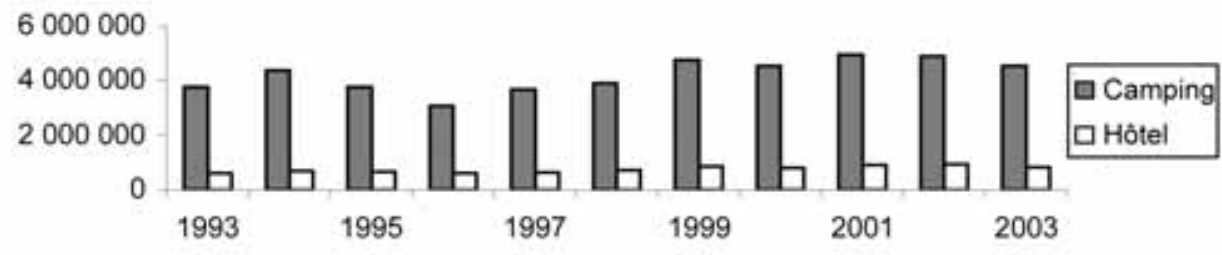

Source : Observatoire régional du tourisme du Languedoc-Roussillon (2004).

destinations comportent des caractéristiques touristiques similaires à la région. De plus, elles attirent les touristes par leur aspect « découverte ".

En ce qui concerne l'analyse de la durée des séjours, nous sommes amenés à faire le rapport entre le nombre de nuitées et le nombre d'arrivées. Différents points peuvent alors être soulignés selon le type d'hébergement. Pour les hôtels, la durée moyenne annuelle de séjour est stable tout au long de la période considérée avec 1,8 nuitée par touriste. Autrement dit, le nombre de nuitées et le nombre d'arrivées varient exactement dans les mêmes proportions. Dans le cas des campings, nous observons une tendance générale légèrement à la hausse, passant de 7,2 à 7,7 nuitées par touriste, soit une augmentation de $7 \%$ sur les dix dernières années. Mais cette tendance est altérée par une baisse sensible dans la seconde moitié des années 1990, où cette durée moyenne des séjours passe sous la barre d'une semaine par touriste $(6,8$ nuitées en 1997 précisément).

C'est le type d'hébergement de plein air qui caractérise la région $\mathrm{L}-\mathrm{R}$, puisque celui-ci, avec beaucoup moins d'arrivées, enregist- re beaucoup plus de nuitées $(7,7$ nuitées contre 1,7 nuitée par touriste pour les hôtels). Les hôtels sont donc bien moins performants. II va sans dire que ces différents résultats justifient la spécialisation de cette région dans les campings : c'est la première région française pour ce type d'hébergement. Cependant, ce résultat n'a rien de surprenant dans la mesure où la région est composée plutôt d'«E-attraction " que de «D-attraction " (Peypoch et Solonandrasana, 2004 ; à paraître). Nous éclaircirons nos propos dans la deuxième partie de notre exposé.

\section{Analyse de la durée des séjours selon la nationalité des touristes}

Comme nous l'avons souligné précédemment, afin de mieux comprendre l'évolution de la durée des séjours, nous allons nous intéresser aux touristes selon leur pays d'origine. Nous considérons alors quatre pays pour les touristes en L-R: I'Allemagne, l'Angleterre, la Belgique et les Pays-Bas. Ce choix est justifié par le fait que ces touristes représentent en moyenne, chaque année, plus de $85 \%$ du nombre total de touristes étrangers dans les campings et les hôtels de la région $L-R$ (Observatoire régional du $L-R, 2004)$. 
Graphique 2a

Évolution des arrivées en hôtel par pays d’origine

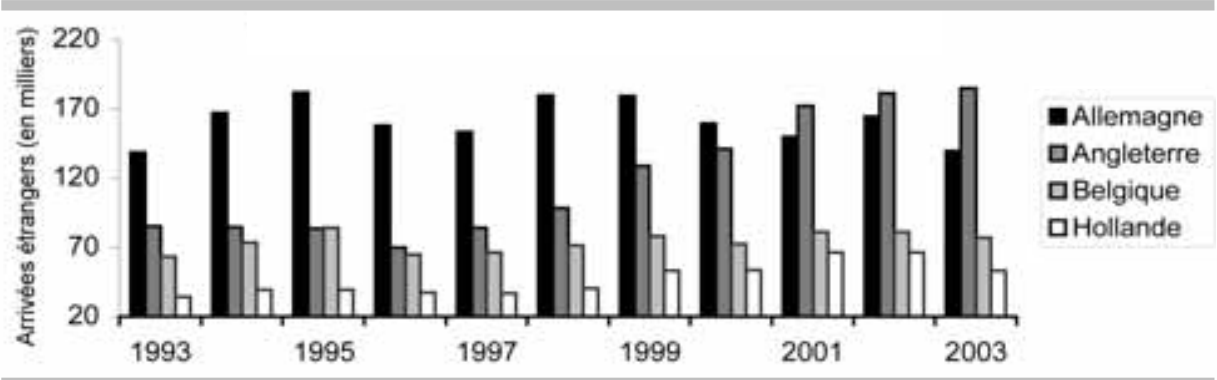

Source : Observatoire régional du tourisme du Languedoc-Roussillon (2004).

\section{Graphique 2b}

Évolution des arrivées en camping par pays d'origine

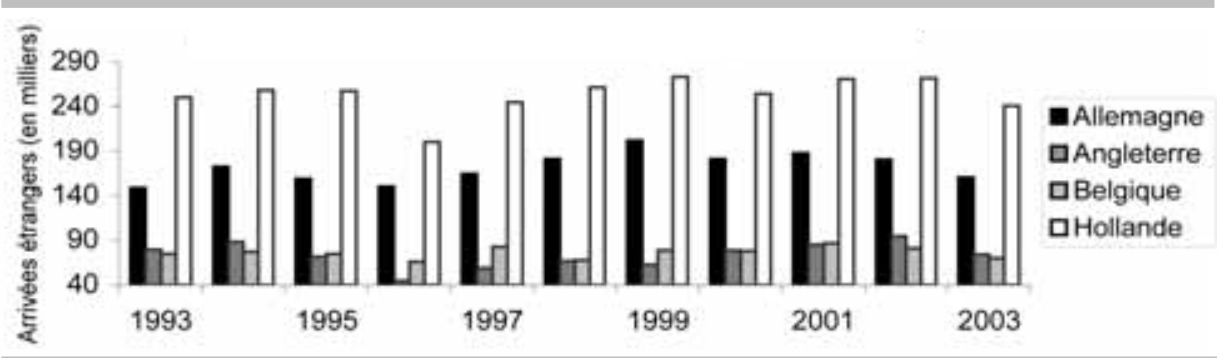

Source : Observatoire régional du tourisme du Languedoc-Roussillon (2004).

\section{Graphique 3a}

\section{Évolution des nuitées en hôtel par pays d'origine}

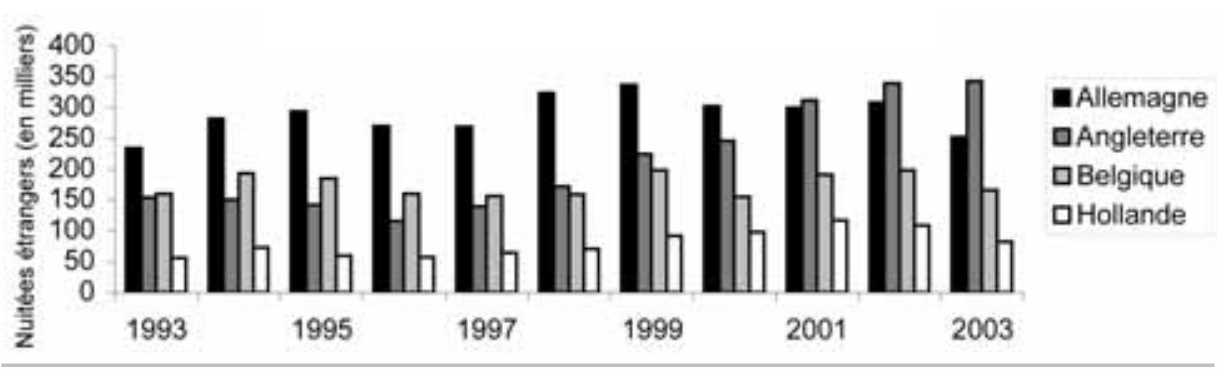

Source : Observatoire régional du tourisme du Languedoc-Roussillon (2004).

\section{Graphique 3b}

\section{Évolution des nuitées en camping par pays d'origine}

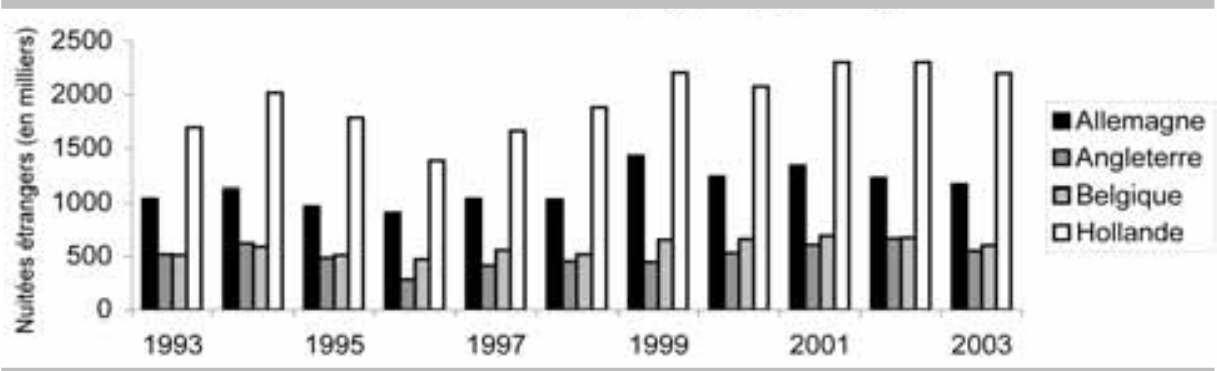

Source : Observatoire régional du tourisme du Languedoc-Roussillon (2004).
La complexité à représenter de manière synthétique l'ensemble des données nécessaires à notre analyse nous amène à séparer les représentations des deux premières variables qui sont le nombre d'arrivées et de nuitées et ainsi à représenter, par les graphiques $2 a$ et $2 b$, les évolutions du nombre d'arrivées selon le pays d'origine des touristes, respectivement en hôtel et en camping.

Les graphiques $2 a$ et $2 b$ ne permettent pas de dégager une tendance commune pour l'ensemble des nationalités de touristes et cela, quel que soit le type d'hébergement considéré. Cependant, ils permettent tout de même de signaler que les touristes allemands et belges se répartissent de manière équitable entre les deux types d'hébergement durant les dix dernières années. Les Hollandais, quant à eux, n'adoptent pas le même comportement selon le type d'hébergement. En effet, ils fréquentent plus les hébergements de plein air que les hôtels. Enfin, le nombre de touristes anglais fréquentant les hôtels a connu une forte hausse depuis 1996, alors que ceux privilégiant l'hébergement de plein air ont vu leur nombre se stabiliser.

De la même manière, les graphiques $3 a$ et $3 b$ présentent les évolutions du nombre de nuitées dans les campings et les hôtels du L-R selon les quatre pays d'origine des touristes cités précédemment.

Comme pour les graphiques $1 \mathrm{a}$ et $1 \mathrm{~b}$, nous constatons que les évolutions du nombre de nuitées selon le type d'hébergement et la nationalité des touristes suivent les mêmes tendances que celles du nombre d'arrivées. Plus précisément, pour l'hébergement en hôtel, la forte augmentation du nombre de nuitées provient surtout des Anglais. Cependant, depuis 2001, une érosion du nombre de nuitées est constatée et touche principalement la clientèle allemande. En ce qui concerne les campings, après des années difficiles dans le milieu des années 1990, le nombre de nuitées a augmenté de 1996 à 2003 et les plus fortes progressions sont à mettre à l'actif des touristes anglais (134\%) et hollandais (67\%).

Lorsque nous mettons en relation le nombre de nuitées et d'arrivées, nous pouvons déterminer comme précédemment la durée moyenne de séjours. Nous constatons alors, d'une part pour les hôtels, une certaine constance pour les touristes allemands et an- 
glais, soit 1,8 nuitée par personne. Le score le plus faible concerne les touristes hollandais (1,5 nuitée par personne), alors que les belges effectuent de loin les séjours les plus longs avec 2,5 nuitées par personne. D'autre part, dans le cas des campings, de faibles variations sont à noter pour les touristes allemands et anglais, avec respectivement 6,9 et 7 nuitées par touriste. Les plus fortes progressions sont attribuées aux touristes belges (de 6,8 nuitées en 1993 à 8,6 nuitées en 2003, soit une augmentation de $27 \%$ ) et hollandais (de 6,8 nuitées en 1993 à 9,1 nuitées en 2003, soit une hausse de $34 \%$ ).

II ressort des précédents résultats que les durées de séjours des touristes belges et hollandais n'ont cessé d'augmenter durant ces dix dernières années. De plus, les touristes belges effectuent les plus longs séjours, quel que soit le type d'hébergement. Cependant, en termes de retombées économiques, ces résultats doivent être analysés avec précaution dans la mesure où ils ne tiennent pas compte des dépenses que les touristes sont susceptibles d'effectuer dans la région.

\section{La relation entre l'attraction touristique et la durée de séjours}

Comme nous l'avons dit en introduction, le $L-R$ est une région qui renferme de nombreux atouts. (Klemm 1996) regroupe ces derniers dans cinq principales zones: ses côtes, son arrière-pays, ses montagnes, ses termes d'attraction et ses villes historiques. Pour éviter d'alourdir notre analyse avec ces différents paramètres, c'est-àdire suivant les différentes activités que les touristes peuvent pratiquer, nous allons utiliser la nouvelle approche de classification de Caccomo et Solonandrasana (2002) qui permet de regrouper les activités en termes d'attraction («E-attraction » ou «D-attraction »). Ainsi, la conception qu'ont différents touristes de la destination va être décrite dans leur comportement, plus précisément, à travers la durée de leur séjour.

\section{La Classification des activités touristiques en «E-attraction » ou «D-attraction »}

On appelle attraction touristique l'élément qui attire le touriste hors de son environnement habituel. Différentes approches permettent de classifier les attractions et sont résumées par
Mehmetoglu et Abelsen (2005). Nous privilégions celle qui place le touriste au cœur de la classification (Caccomo et Solonandrasana, 2002). Cette démarche offre une vision globale et ne se contente pas d'identifier l'attraction d'un point de vue purement géographique, socioéconomique ou managérial. En effet, ces auteurs ont récemment mis l'accent, d'une part, sur le fait que l'unité de mesure de tout produit touristique est le temps ; ainsi, l'évaluation de la satisfaction du touriste dépend de la durée de son séjour. D'autre part, toutes les attractions peuvent être regroupées en seulement deux types : les attractions dites "découvertes", appelées "D-attraction », ou les attractions dites «évasions », appelées «E-attraction». C'est le touriste qui détermine, en la consommant, la nature de l'attraction. La destination sera à prédominance D-attraction à ses yeux s'il la consomme de manière passive dans la mesure où il ne s'apparente qu'à un simple spectateur. En d'autres termes, il se contente de contempler le produit durant son séjour. Une fois découverte, la destination ne présente plus aucun intérêt pour le touriste qui voit sa satisfaction décroître si son séjour se prolonge. En revanche, la destination sera à prédominance E-attraction aux yeux du touriste s'il la consomme de manière active, dans la mesure où il participe pleinement à son exploration; dans ce cas, il s'apparente à un acteur car il participe activement au déroulement du motif de son séjour. Pour le touriste, la destination présente un attrait permanent et sa satisfaction croît (ou au moins se maintient) au fur et à mesure qu'il explore cette dernière sans atteindre de point de retournement.

Peypoch et Solonandrasana (2004, à paraître), dans leurs travaux, ont prolongé ces réflexions. Ils ont pu montrer à l'aide d'un modèle économétrique que la notion d'E-attraction est liée à celle de la répétition ou du prolongement de la consommation. Étant donné que la satisfaction du touriste augmente avec la durée du séjour, elle n'atteindra jamais de point de retournement ; elle atteindra à la limite un maximum auquel elle se maintiendra tout le long du séjour. De plus, en suivant Peypoch et al. (2005), nous pouvons conclure qu'il y aura un effet d'accumulation. Cependant, la D-attraction caractérise un comportement différent de la part du touriste, dans la mesure où il n'y a plus de répétition dans la consommation. La satisfaction du touriste atteint plus ou moins rapidement un maximum, c'est-à-dire un seuil de saturation, pour ensuite diminuer considérablement. Nous mesurons donc toute l'importance de ces deux notions afin de caractériser la durée des séjours. En effet, une répétition ou un prolongement de la consommation augmentera la durée de séjour et caractérisera une E-attraction aux yeux des touristes, alors que, dans le cas contraire, la durée de séjour sera plus courte et donc synonyme d'une D-attraction pour le touriste.

\section{Analyse des séjours touristiques en termes d'attraction}

En utilisant le découpage de Klemm (1996) en cinq zones d'activités, la zone représentant les sites historiques est celle qui accueille le moins de visiteurs, loin derrière les côtes et l'arrière-pays. En effet, en 2003, ces derniers représentent respectivement $68 \%$ et $18 \%$ des séjours touristiques, alors que les sites historiques représentent à peine $3 \%$. Ce faible score est expliqué par une plus ou moins rareté de ces sites. Visiter ou découvrir la région peut, par conséquent, se faire en peu de temps. Lorsque nous considérons ces différentes notions, nous pouvons parvenir aux interprétations suivantes. D'une manière générale, quel que soit le type d'hébergement, les touristes belges effectuent de très longs séjours en LanguedocRoussillon. Ils considèrent donc que la région est une destination à dominance E-attraction, c'est-à-dire qu'ils consomment les attractions de la région de façon active. Ces touristes ne cherchent pas explicitement à découvrir un site en particulier ou à visiter un monument spécifique. Par conséquent, leur satisfaction, pour une période relativement courte, n'atteint pas un seuil de saturation. En d'autres termes, elle augmente avec la durée de séjour, ce qui les conduit à adopter un comportement de consommation répétitif. Cela est renforcé par le fait que, selon l'enquête Eurêma ${ }^{3}, 70$ \% des Belges viennent dans la région pour pratiquer le repos et la baignade. II en est de même pour les touristes hollandais dont la durée des séjours a augmenté de $34 \%$ durant ces dix dernières années et qui sont $85 \%$ à choisir cette destination pour les mêmes motifs. Au contraire, les touristes allemands effectuent des séjours plus courts dont la durée est restée stable par rapport à 1993. II paraît alors évident que leur comportement et leur motivation sont tout autres, ce que confirme l'enquête Eurêma qui indique, par exemple, que les Allemands ne sont que de $20 \%$ à $30 \%$ à rechercher le repos et la mer, 
alors qu'ils sont environ $40 \%$ à rechercher une activité culturelle pendant le séjour. Leurs comportements indiquent qu'ils recherchent plutôt une D-attraction, puisqu'il n'y a clairement pas d'effet de répétition dans leur consommation et la durée de leurs séjours est en conséquence beaucoup plus courte. Quant aux touristes anglais, la durée de leurs séjours n'a augmenté que de $15 \%$ en dix ans et reste assez faible ; leurs comportements sont donc plus difficilement identifiables, ce qui s'apparente à la recherche d'un mélange entre les deux types d'«attraction ».

De plus, pour toutes les nationalités de touristes, il apparaît clairement que la durée de séjours en camping est nettement supérieure à celle en hôtel. Par conséquent, nous pouvons également établir une classification des touristes en termes d' " attraction » suivant le type d'hébergement choisi. Ainsi, les touristes qui fréquentent les hébergements de plein air semblent considérer la région comme une E-attraction, alors que ceux qui séjournent en hôtel y voient plutôt une D-attraction. En effet, les touristes qui privilégient l'hébergement de plein air ont un comportement répétitif dans leur consommation, qui allonge la durée des séjours. II convient également de noter que ce type d'hébergement est souvent considéré par les touristes comme une attraction à part entière, mais pas seulement comme un lieu dans lequel on séjourne. II n'est pas rare de rencontrer des touristes qui viennent dans la région pour un motif tel que «se retrouver entre amis au camping et partager des beaux moments ensemble». En revanche, la durée des séjours dans les hôtels est plus courte car les touristes y consomment différemment et certainement pas de manière prolongée ou répétitive. Cette distinction, qui est très nette dans notre cadre d'analyse, à savoir la région LanguedocRoussillon, mérite d'être approfondie dans de futures recherches. Nous croyons qu'il serait intéressant de considérer d'autres destinations touristiques afin de vérifier si, d'une manière générale, le type d'hébergement peut permettre de classifier la prédominance en termes d'«attraction» du comportement des touristes.

\section{Conclusion}

Cet article a tenté de dresser une analyse de la durée des séjours des touristes européens en Languedoc-Roussillon, par le biais de dif- férents paramètres, à savoir : les types d'hébergement (hôtel et camping) et la nationalité des touristes (Allemands, Anglais, Belges et Hollandais). L'analyse par type d'hébergement a permis de mettre en lumière le retard qu'enregistrent les hôtels par rapport aux hébergements de plein air, ainsi que la tendance récente à la baisse du nombre d'arrivées et de nuitées. Le découpage par nationalité, quant à lui, a mis en évidence les différences dans les comportements des touristes. II en ressort que la région $L-R$ attire de plus en plus les touristes anglais, fidélise les touristes belges et hollandais et intéresse moyennement les touristes allemands. Le recoupement de ces différentes analyses a permis de donner une interprétation en termes d' " attraction touristique ». La majorité des touristes européens considèrent que la région est à prédominance E-attraction.

Le fait que la région soit classée par les touristes comme une E-attraction est favorable à un prolongement de la durée des séjours et/ou à une répétition de ces derniers (Peypoch et Solonandrasana, à paraître). L'importance en termes de retombées économiques de l'industrie du tourisme est non négligeable pour la région du Languedoc-Roussillon qui peut améliorer son économie grâce à cette industrie. En effet, par l'intermédiaire des arrivées de touristes et donc des nuitées effectuées, les dépenses touristiques constituent une source de revenu importante. II faut à présent orienter au mieux les différentes politiques touristiques. Pour ce faire, le comportement des touristes doit être pris en compte afin de tirer profit au mieux des différents flux touristiques. Enfin, si nous prenons en compte les derniers résultats de l'Observatoire régional du tourisme (2004) sur la propension à dépenser des touristes par nationalité, la région devrait continuer à attirer les touristes anglais et à conserver les touristes belges, puisqu'ils peuvent dépenser respectivement au-delà de 85 euros et de 60 euros par jour et par touriste.

Nicolas Peypoch, est attaché temporaire d'enseignement et de recherche en sciences économiques, à l'Université de Perpignan.

\section{Bernardin Solonandrasana est maitre de} conférences en sciences économiques, à I'Université de Perpignan.

\section{Notes}

1 Université de Perpignan, Groupe d'études et de recherche en économie mathématique (GEREM), Département des sciences économiques et de gestion.

Les auteurs tiennent à remercier l'Observatoire régional du tourisme du LanguedocRoussillon pour les données qu'ils ont fournies.

2 Toutes les données de ce paragraphe proviennent de l'Observatoire régional du tourisme du Languedoc-Roussillon (2004).

3 Cette enquête de l'Observatoire régional du tourisme en Languedoc-Roussillon fournit le profil des dépenses et des motifs de séjour des touristes selon leur nationalité.

\section{Bibliographie}

Bordet, C., N. Peypoch, et B. Solonandrasana (2004), Influence de l'Espagne sur la fréquentation touristique en Languedoc-Roussillon, Mimeo.

Caccomo, J.-L., et B. Solonandrasana (2001), Innovation dans l'industrie touristique: Enjeux et stratégies, L'Harmattan, Paris, 156 p.

Caccomo, J.-L., et B. Solonandrasana (2002), "Réflexions autour du concept de l'attraction touristique : Analyse et taxonomie ", Téoros, vol. $21, n^{\circ} 3$, p. 68-71.

Institut national de la statistique et des études économiques - INSEE (2004), Repères, Chiffres pour l'économie du L-R, $n^{\circ} 3$.

Klemm, M-S. (1996), "Languedoc Roussillon: Adapting the Strategy ", Tourism Management, vol. 17, n² 2, p. 133-147.

Mehmetoglu, M., et B. Abelsen (2005), «Examining the Visitor Attraction Product ", Tourism Analysis, vol. 9, n 4, p. 269-284.

Observatoire régional du tourisme du LanguedocRoussillon (2000), Enquête "Eurêma ".

Observatoire régional du tourisme du LanguedocRoussillon (2004), Les clientèles du Languedoc-Roussillon.

Peypoch, N., E. Robinot, et B. Solonandrasana (2005), "Which Sustainable Development Perspectives for E-attraction Destination : An Overview of the Economic Impacts », Tourism and Hospitality Planning \& Development, vol. 2, n³, p. 207-212.

Peypoch, N., et B. Solonandrasana (2004), «Une approche économétrique de la fréquentation touristique en Languedoc-Roussillon: Le cas des touristes anglais et belges ", Revue de l'Économie Méridionale, vol. 52, $n^{\circ}$ 205-206, p. 117-129.

Peypoch, N., et B. Solonandrasana (à paraître), «On E-attraction Tourism Destination : Extension and Application ", Theoretical Advances in Tourism Economics, Springer-Verlag.

Racine, P. (1980) «La Mission impossible », Midi Libre, Montpellier. 